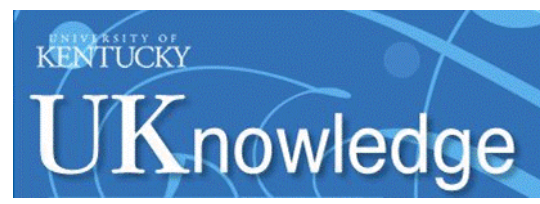

University of Kentucky

UKnowledge

3-1-2012

\title{
Ion-By-Ion Cooling Efficiencies
}

Orly Gnat

California Institute of Technology

Gary J. Ferland

University of Kentucky, gary@uky.edu

Follow this and additional works at: https://uknowledge.uky.edu/physastron_facpub

Part of the Astrophysics and Astronomy Commons, and the Physics Commons

Right click to open a feedback form in a new tab to let us know how this document benefits you.

\section{Repository Citation}

Gnat, Orly and Ferland, Gary J., "Ion-By-lon Cooling Efficiencies" (2012). Physics and Astronomy Faculty Publications. 136.

https://uknowledge.uky.edu/physastron_facpub/136

This Article is brought to you for free and open access by the Physics and Astronomy at UKnowledge. It has been accepted for inclusion in Physics and Astronomy Faculty Publications by an authorized administrator of UKnowledge. For more information, please contact UKnowledge@lsv.uky.edu. 


\section{Ion-By-Ion Cooling Efficiencies}

Digital Object Identifier (DOI)

https://doi.org/10.1088/0067-0049/199/1/20

\section{Notes/Citation Information}

Published in The Astrophysical Journal Supplement Series, v. 199, no. 1, 20, p. 1-5.

(c) 2012. The American Astronomical Society. All rights reserved. Printed in the U.S.A.

The copyright holder has granted permission for posting the article here. 


\title{
ION-BY-ION COOLING EFFICIENCIES
}

\author{
Orly Gnat ${ }^{1,3}$ and Gary J. FerLand ${ }^{2}$ \\ ${ }^{1}$ Theoretical Astrophysics, California Institute of Technology, MC 350-17, Pasadena, CA 91125, USA, and Racah Institute of Physics, \\ The Hebrew University, 91904 Jerusalem, Israel; orlyg@tapir.caltech.edu \\ 2 Department of Physics and Astronomy, University of Kentucky, Lexington, KY 40506, USA \\ Received 2010 October 11; accepted 2012 January 4; published 2012 March 1
}

\begin{abstract}
We present ion-by-ion cooling efficiencies for low-density gas. We use Cloudy (version 10.00) to estimate the cooling efficiencies for each ion of the first 30 elements $(\mathrm{H}-\mathrm{Zn})$ individually. We present results for gas temperatures between $10^{4}$ and $10^{8} \mathrm{~K}$, assuming low densities and optically thin conditions. When nonequilibrium ionization plays a significant role the ionization states deviate from those that obtain in collisional ionization equilibrium (CIE), and the local cooling efficiency at any given temperature depends on specific nonequilibrium ion fractions. The results presented here allow for an efficient estimate of the total cooling efficiency for any ionic composition. We also list the elemental cooling efficiencies assuming CIE conditions. These can be used to construct CIE cooling efficiencies for non-solar abundance ratios or to estimate the cooling due to elements not included in any nonequilibrium computation. All the computational results are listed in convenient online tables.
\end{abstract}

Key words: atomic processes - ISM: general - plasmas

Online-only material: color figure, supplemental data file (tar.gz)

\section{INTRODUCTION}

The radiative cooling efficiencies ${ }^{4}$ of hot $\left(10^{4}-10^{8} \mathrm{~K}\right)$ lowdensity gas are important quantities in the study of the diffuse interstellar and intergalactic medium. They determine the thermal and dynamical properties and evolution in a variety of astrophysical systems, ranging from local interstellar clouds to shocks in intergalactic filaments.

Computations of hot gas cooling efficiencies assuming collisional ionization equilibrium (CIE) have been studied extensively (House 1964; Tucker \& Gould 1966; Allen \& Dupree 1969; Cox \& Tucker 1969; Jordan 1969; Raymond et al. 1976; Shull \& van Steenberg 1982; Gaetz \& Salpeter 1983; Arnaud \& Rothenflug 1985; Boehringer \& Hensler 1989; Sutherland \& Dopita 1993; Landi \& Landini 1999; Benjamin et al. 2001). These CIE cooling efficiencies depend only on the gas temperature and metallicity. However, there are many cases for which CIE conditions do not apply. For example, nonequilibrium ionization is bound to occur when an initially hot gas cools radiatively below $\sim 10^{6} \mathrm{~K}$ (Kafatos 1973; Shapiro \& Moore 1976; Edgar \& Chevalier 1986; Schmutzler \& Tscharnuter 1993; Sutherland \& Dopita 1993; Smith et al. 1996; Gnat \& Sternberg 2007). Below this temperature, cooling becomes rapid compared to electron-ion recombinations, and the gas at any temperature tends to remain "overionized" compared to gas in CIE. In conduction fronts surrounding evaporating clouds (e.g., Borkowski et al. 1990; Gnat et al. 2010), nonequilibrium ionization occurs when the ionization time is long compared to the rate of temperature change. In this case, the gas tends to remain underionized compared to CIE. Nonequilibrium ionization also plays a role

\footnotetext{
3 Chandra Fellow.

4 Here we refer to $\Lambda$, with units erg $\mathrm{cm}^{3} \mathrm{~s}^{-1}$. There is no standard term for $\Lambda$ in the literature. We use the term "cooling efficiency" (e.g., Dalgarno \& McCray 1972). Other common terms include "cooling coefficient" (e.g., Benjamin et al. 2001), "energy loss function" (e.g., Shapiro \& Moore 1976), "plasma emissivity" or "contribution function" (e.g., Landi \& Landini 1999), "cooling function" or "cooling rate" (e.g., Sutherland \& Dopita 1993), and "emissivity coefficient" (e.g., Graney \& Sarazin 1990). The variety of names in part reflects differences in the book-keeping scheme used to follow the cooling (see Section 2).
}

in fast radiative shock waves (e.g., Allen et al. 2008; Gnat \& Sternberg 2009) and in turbulent mixing layers (e.g., Slavin et al. 1993).

When departures from CIE are significant, the cooling efficiencies are no longer a function of just the gas temperature and metallicity, but instead depend on the specific time-dependent ion fractions. The use of convenient tables with known CIE cooling efficiencies must be replaced with a detailed computation of the nonequilibrium cooling, taking into account all the relevant microphysical processes which include numerous emission lines, thermal bremsstrahlung, and ionization and recombination cooling. This requires the collection of a large set of atomic data for all the relevant processes and is computationally complex compared with using look-up tables.

Recently, first attempts have been made at including nonequilibrium ionization physics in large-scale hydrodynamical simulations, with applications for both cosmological metal absorption through the warm/hot intergalactic medium (e.g., Cen \& Ostriker 2006; Cen \& Fang 2006) and emission lines from galaxy clusters (e.g., Akahori \& Yoshikawa 2010). Because of the complexity of nonequilibrium cooling, such simulations have so far not included self-consistent nonequilibrium cooling efficiencies. It is simpler to include nonequilibrium cooling in hydrodynamical simulations by using look-up tables for the nonequilibrium cooling rate (e.g., Oppenheimer \& Davé 2009). However, in these simplified models, the nonequilibrium cooling rates are not consistent with the assumed ion fractions.

In this paper, we present ion-by-ion cooling efficiencies. We list the cooling efficiencies for each ion of the first 30 elements $(\mathrm{H}-\mathrm{Zn})$ individually. We present results for gas temperatures between $10^{4}$ and $10^{8} \mathrm{~K}$, assuming optically thin, low-density conditions. The sum of ionic cooling efficiencies, weighted by the nonequilibrium ion densities, then provides an efficient-to-compute and self-consistent nonequilibrium cooling efficiency. We also list the elemental cooling efficiencies assuming CIE conditions. These can be used to construct CIE cooling efficiencies for non-solar abundance ratios or to estimate the remaining cooling due to elements not included in any timedependent computation. 
This paper is not a new calculation of the cooling functions (cf. Schure et al. 2009). We do not present any new atomic data. Instead, we list the current cooling functions included in Cloudy (version 10.00) in convenient online tables that are useful in any numerical computation in which the ion abundances are not in photoionization or collisional equilibrium. It is the first time that the individual ionic cooling efficiencies are listed in an accessible format. As we describe below, the new framework that we present here will be periodically maintained and updated as improved atomic data become available.

The outline of this paper is as follows. In Section 2, we describe the computational method. In Section 3, we present the results for the ion-by-ion cooling efficiencies, and for the element-by-element CIE cooling efficiencies. We summarize in Section 4.

\section{METHOD}

We used Cloudy (version 10.00; Ferland et al. 1998) to compute the ion-by-ion cooling efficiencies of every ion of the first 30 elements $(\mathrm{H}-\mathrm{Zn})$. All the cooling processes considered by Cloudy are described in detail in Osterbrock \& Ferland (2006), and include collisional excitations followed by line emission, recombinations with ions, collisional ionizations, and thermal bremsstrahlung. ${ }^{5}$ The electron cooling efficiency includes the removal of electron kinetic energy via recombinations with ions, collisional ionizations, collisional excitations followed by prompt line emissions, and thermal bremsstrahlung. ${ }^{6}$ Cloudy does not include the ionization potential energies as part of the total internal energy, but instead follows the loss and gain of the electron kinetic energy only. Therefore, while the energy of each recombination photon is the sum of the electron kinetic energy and the binding energy of the ion, in the definition of the cooling (see Osterbrock \& Ferland 2006; Gnat \& Sternberg 2007) the ionization potential energy that is included in the recombination radiation does not appear. Only the kinetic energy of the recombining electrons contributes to the cooling efficiency. On the other hand, kinetic energy removed via collisional ionization is included in the cooling. If ionization potential energy is considered as part of the total internal energy, then collisional ionization does not lead to a net energy loss, since the kinetic energy removed is merely stored as potential energy. Either way of accounting for the energy losses leads to the same net (i.e., cooling minus heating) cooling efficiency.

For each ionization state $i$ of each element $E$, we constructed a series of models for different gas temperature between $10^{4}$ and $10^{8} \mathrm{~K}$. Each model includes only hydrogen and the element $E$. The abundances of all other elements are set to zero. We set an electron density $n_{e}=1 \mathrm{~cm}^{-3}$, regardless of composition and ionization state. We define the ionization states of the element $E$ so that the fractional abundance of the species $E_{i}$ is 1 , and the abundances of all other ions are 0 . We further set the abundance of element $E$ to be $10^{15}$ larger than that of hydrogen, so that $n_{\mathrm{H}}=10^{-15} \mathrm{~cm}^{-3}$ and $n\left(E_{i}\right)=1 \mathrm{~cm}^{-3}$. Effectively, each such model contains only the species $E_{i}$ and free electrons at the specified electron

\footnotetext{
5 Cloudy is available at http://www.nublado.org/. The code documentation including a full description of all cooling processes is available at http://viewvc.nublado.org/index.cgi/tags/release/c08.00/-docs/?root=cloudy (and will be described in G. J. Ferland et al. 2012, in preparation). To get the full list of references to the atomic data in this version, see instructions in Section 13.5 of Cloudy's third volume of documentation (Hazy3_08.pdf).

6 The cooling is always computed with respect to the source ion (e.g., recombinations are attributed to the more ionized species).
}

Table 1

Ion-by-ion Cooling Efficiencies

\begin{tabular}{lccc}
\hline \hline$T$ & $\begin{array}{c}\Lambda_{e, \mathrm{HI}}(\mathrm{HI}) \\
\left(\mathrm{erg} \mathrm{cm} \mathrm{cm}^{-1}\right)\end{array}$ & $\begin{array}{c}\Lambda_{e, \mathrm{H} \mathrm{II}}(\mathrm{H} \mathrm{II}) \\
\left(\mathrm{erg} \mathrm{cm}^{3} \mathrm{~s}^{-1}\right)\end{array}$ & $\begin{array}{c}\Lambda_{e, \mathrm{H}}(\mathrm{H} \text { at CIE }) \\
\left(\mathrm{erg} \mathrm{cm}^{3} \mathrm{~s}^{-1}\right)\end{array}$ \\
\hline $1.00 \times 10^{4}$ & $4.59 \times 10^{-24}$ & $6.26 \times 10^{-25}$ & $4.58 \times 10^{-24}$ \\
$1.05 \times 10^{4}$ & $7.93 \times 10^{-24}$ & $6.36 \times 10^{-25}$ & $7.90 \times 10^{-24}$ \\
$1.10 \times 10^{4}$ & $1.33 \times 10^{-23}$ & $6.46 \times 10^{-25}$ & $1.32 \times 10^{-23}$ \\
\hline
\end{tabular}

Notes. The full table (available in the online version as a supplemental data file) lists the ion-by-ion cooling efficiencies for all the different ions of the first 30 elements $(\mathrm{H}-\mathrm{Zn})$, and for the elemental cooling efficiencies assuming CIE. For a guide see Table 2 .

temperature. ${ }^{7}$ Note that because we force the electron density $\left(n_{e}=1 \mathrm{~cm}^{-3}\right)$, the resulting cooling efficiencies only apply when excitations by thermal electrons dominate over neutral impact excitations. When neutral excitations are significant (typically at low temperatures and low electron fractions, $n_{e} / n_{\mathrm{H}}$ ) these efficiencies will underestimate the cooling. This scheme also does not capture cooling due to charge transfer reactions.

For each element, we also compute the cooling efficiencies assuming CIE ion fractions. In these models, we set the abundance of the element $E$ to be $10^{15}$ larger than that of hydrogen (so that again $n_{\mathrm{H}}=10^{-15} \mathrm{~cm}^{-3}$ and $n(E)=1 \mathrm{~cm}^{-3}$ ), and we force an electron density $n_{e}=1 \mathrm{~cm}^{-3}$. We allow Cloudy to compute the CIE ion fractions $x\left(E_{i}\right)$. For each element $E$, the ion fractions $x\left(E_{i}\right)=n\left(E_{i}\right) / n(E)$ must at all times satisfy

$$
\sum_{i} x\left(E_{i}\right)=1
$$

where $n\left(E_{i}\right)$ is the density $\left(\mathrm{cm}^{-3}\right)$ of ions in ionization stage $i$ of element $E, n(E)=n_{\mathrm{H}} A_{E}, n_{\mathrm{H}}$ is the total hydrogen density, and $A_{E}$ is the abundance of element $E$ relative to hydrogen. These models yield the total cooling efficiency due to the CIE ion distribution of element $E$.

The tables presented in this paper provide easy access to different components of the total cooling function computed in version 10.00 of Cloudy. The cooling functions included in Cloudy are constantly being updated as improved data become available. We will update this table, keeping its current format, to provide ready access to these future calculations (see http://wise-obs.tau.ac.il/ orlyg/ion_by_ion/). This way, codes that can parse the current tables can be easily updated as better atomic data become available.

\section{ION-BY-ION COOLING EFFICIENCIES}

We have carried out computations of the cooling efficiencies $\Lambda_{e, \text { ion }}(T)$ for each ion of the first 30 elements, $\mathrm{H}-\mathrm{Zn}$ (with atomic numbers 1-30), as a function of temperature. The results are listed in tabular form in Table 1. The full table is available as a supplemental data file in the online version and is divided into lettered parts $\mathrm{A}-\mathrm{AD}$, as is outlined in Table 2. For each element $E$ with atomic number $Z$, the first column in Table 1 lists the temperature, and the next $Z+1$ columns list the cooling efficiencies, $\Lambda_{e, \text { ion }}\left(\mathrm{erg} \mathrm{s}^{-1} \mathrm{~cm}^{3}\right)$ for the different ionization states, starting with the neutral atom, and ending with the fully stripped ion. The cooling rate per unit volume due to ionization state $i$ of element $E$ is then given by

\footnotetext{
7 We verify that the contribution of hydrogen to each model is negligible by changing the abundance of the relevant species from $10^{15}$ times $n_{\mathrm{H}}$ to $10^{10}$ times $n_{\mathrm{H}}$ and verifying that the results remain unaltered.
} 
Table 2

Cooling Data and Solar Elemental Abundances

\begin{tabular}{|c|c|c|c|c|}
\hline$Z$ & Element & Table & Abundance $(\mathrm{X} / \mathrm{H})_{\odot}$ & References \\
\hline 1 & Hydrogen & $1 \mathrm{~A}$ & 1 & \\
\hline 2 & Helium & $1 \mathrm{~B}$ & $8.33 \times 10^{-2}$ & B \\
\hline 3 & Lithium & $1 \mathrm{C}$ & $2.04 \times 10^{-9}$ & $\mathrm{C}$ \\
\hline 4 & Beryllium & $1 \mathrm{D}$ & $2.63 \times 10^{-11}$ & $\mathrm{C}$ \\
\hline 5 & Boron & $1 \mathrm{E}$ & $6.17 \times 10^{-10}$ & $\mathrm{C}$ \\
\hline 6 & Carbon & $1 \mathrm{~F}$ & $2.45 \times 10^{-4}$ & A \\
\hline 7 & Nitrogen & $1 \mathrm{G}$ & $6.03 \times 10^{-5}$ & A \\
\hline 8 & Oxygen & $1 \mathrm{H}$ & $4.57 \times 10^{-4}$ & A \\
\hline 9 & Fluorine & $1 \mathrm{I}$ & $3.02 \times 10^{-8}$ & $\mathrm{C}$ \\
\hline 10 & Neon & $1 \mathrm{~J}$ & $1.95 \times 10^{-4}$ & DT \\
\hline 11 & Sodium & $1 \mathrm{~K}$ & $2.14 \times 10^{-6}$ & $\mathrm{C}$ \\
\hline 12 & Magnesium & $1 \mathrm{~L}$ & $3.39 \times 10^{-5}$ & A \\
\hline 13 & Aluminum & $1 \mathrm{M}$ & $2.95 \times 10^{-6}$ & $\mathrm{C}$ \\
\hline 14 & Silicon & $1 \mathrm{~N}$ & $3.24 \times 10^{-5}$ & A \\
\hline 15 & Phosphorus & 10 & $3.20 \times 10^{-7}$ & $\mathrm{C}$ \\
\hline 16 & Sulfur & $1 \mathrm{P}$ & $1.38 \times 10^{-5}$ & A \\
\hline 17 & Chlorine & $1 Q$ & $1.91 \times 10^{-7}$ & $\mathrm{C}$ \\
\hline 18 & Argon & $1 \mathrm{R}$ & $2.51 \times 10^{-6}$ & $\mathrm{C}$ \\
\hline 19 & Potassium & $1 \mathrm{~S}$ & $1.32 \times 10^{-7}$ & $\mathrm{C}$ \\
\hline 20 & Calcium & $1 \mathrm{~T}$ & $2.29 \times 10^{-6}$ & $\mathrm{C}$ \\
\hline 21 & Scandium & $1 \mathrm{U}$ & $1.48 \times 10^{-9}$ & $\mathrm{C}$ \\
\hline 22 & Titanium & $1 \mathrm{~V}$ & $1.05 \times 10^{-7}$ & $\mathrm{C}$ \\
\hline 23 & Vanadium & $1 \mathrm{~W}$ & $1.08 \times 10^{-8}$ & $\mathrm{C}$ \\
\hline 24 & Chromium & $1 X$ & $4.68 \times 10^{-7}$ & $\mathrm{C}$ \\
\hline 25 & Manganese & $1 Y$ & $2.88 \times 10^{-7}$ & $\mathrm{C}$ \\
\hline 26 & Iron & $1 Z$ & $2.82 \times 10^{-5}$ & A \\
\hline 27 & Cobalt & $1 \mathrm{AA}$ & $8.32 \times 10^{-8}$ & $\mathrm{C}$ \\
\hline 28 & Nickel & $1 \mathrm{AB}$ & $1.78 \times 10^{-6}$ & $\mathrm{C}$ \\
\hline 29 & Copper & $1 \mathrm{AC}$ & $1.62 \times 10^{-8}$ & $\mathrm{C}$ \\
\hline 30 & Zinc & $1 \mathrm{AD}$ & $3.98 \times 10^{-8}$ & $\mathrm{C}$ \\
\hline
\end{tabular}

References. (A) Asplund et al. 2005; (B) Ballantyne et al. 2000; (C) adopted from Cloudy, based on Grevesse \& Sauval 1998; (DT) Drake \& Testa 2005.

$n$ (ion) $n_{e} \Lambda_{e, \text { ion }}\left(\mathrm{erg} \mathrm{s}^{-1} \mathrm{~cm}^{-3}\right)$. For example, Table $1 \mathrm{~A}$ shows that the cooling rate due to neutral hydrogen at a temperature of $1.1 \times 10^{4} \mathrm{~K}$ is $1.33 \times 10^{-23} n\left(\mathrm{H}^{0}\right) n_{e}$ erg s$^{-1} \mathrm{~cm}^{-3}$ whereas the cooling efficiency due to ionized hydrogen at the same temperature is $6.47 \times 10^{-25} n\left(\mathrm{H}^{+}\right) n_{e} \mathrm{erg} \mathrm{s}^{-1} \mathrm{~cm}^{-3}$.

Figure 1 shows the ion-by-ion cooling efficiencies for hydrogen (upper panel) and for helium (lower panel). This figure confirms that if neutral hydrogen exists at high temperatures, it cools orders of magnitude more efficiently than ionized hydrogen. For example, at a temperature of $10^{6} \mathrm{~K}$, neutral hydrogen has a cooling efficiency of $\sim 10^{-18} \mathrm{erg} \mathrm{cm}^{3} \mathrm{~s}^{-1}$, due to collisional ionizations and $\operatorname{Ly} \alpha$ emission. Ionized hydrogen has a cooling efficiency of $\sim 2 \times 10^{-24} \mathrm{erg} \mathrm{cm}^{3} \mathrm{~s}^{-1}$, due to thermal bremsstrahlung emission.

The total cooling due to a specific element depends on the ion abundances. Figure 2 shows the cooling efficiencies versus temperature for the different carbon ions (see Table 1F). The top panel shows the ion-by-ion cooling efficiencies, $\Lambda_{e}$, ion. These can be used to compute the total carbon cooling efficiency for any composition. As an example, in the middle panel we show the carbon CIE ion fractions. The bottom panel then shows the contribution of each ion to the CIE carbon cooling efficiency $\Lambda_{e, \mathrm{C}}($ ion $)=x_{\text {ion }} \Lambda_{e, \text { ion. }}$. For example, at a given carbon density $n(\mathrm{C})$, the contribution of $\mathrm{C}^{3+}$ to the CIE cooling rate per volume is $n(\mathrm{C}) n_{e} \Lambda_{e, \mathrm{C}}\left(\mathrm{C}^{3+}\right) \mathrm{erg} \mathrm{s}^{-1} \mathrm{~cm}^{-3}$. The sum of CIE cooling efficiencies over all carbon ions is shown by the thick gray curve in the lower panel. The carbon CIE cooling efficiency has two peaks. The first peak, at $6 \times 10^{4}-2 \times 10^{5} \mathrm{~K}$, is due to $\mathrm{C}^{+}$,

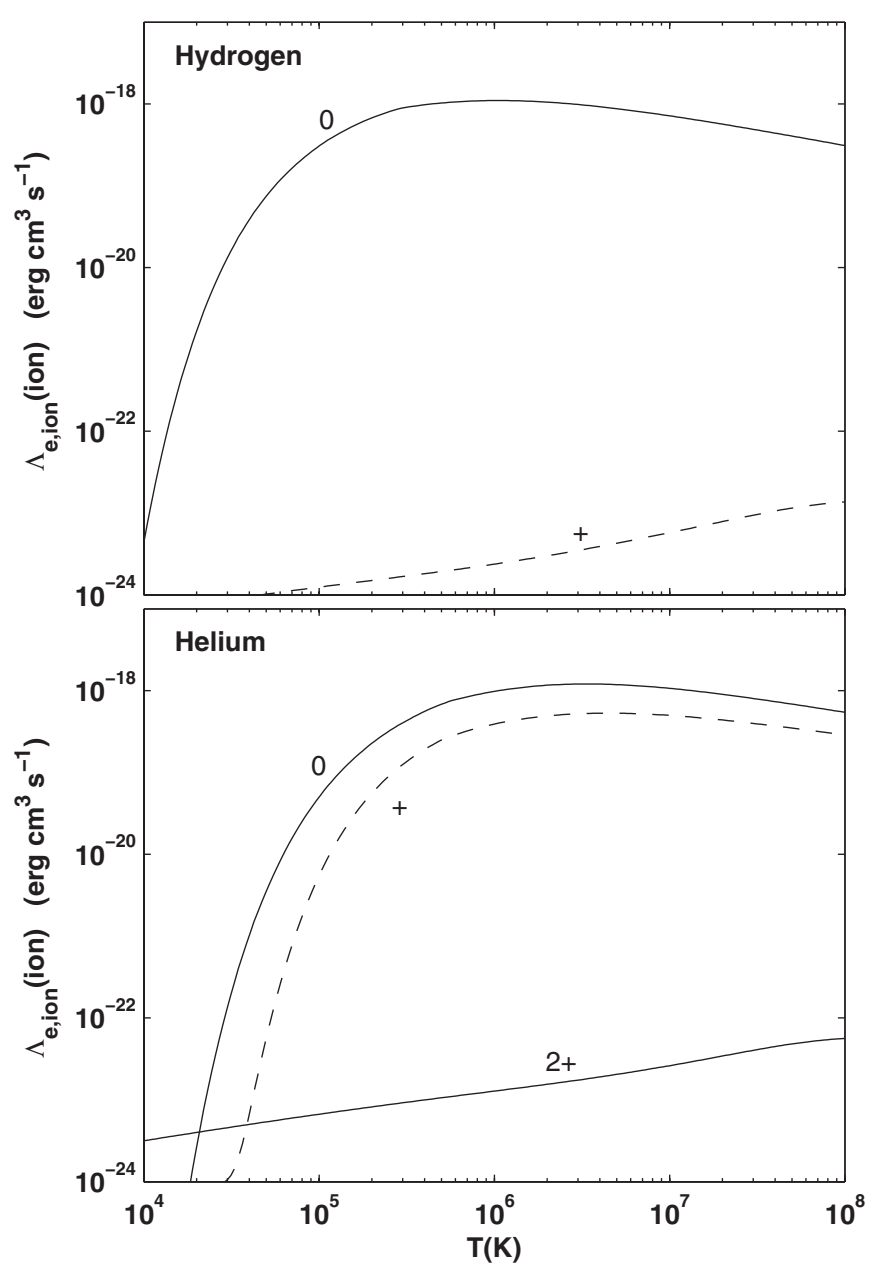

Figure 1. Ion-by-ion cooling efficiencies for hydrogen ions (upper panel) and for helium ions (lower panel).

$\mathrm{C}^{2+}$, and $\mathrm{C}^{3+}$. These ions are responsible for the familiar carbon peak in the solar metallicity CIE cooling curve at $\sim 10^{5} \mathrm{~K}$ (see Section 3.1). The second peak, at $\sim 10^{6} \mathrm{~K}$, is due to $\mathrm{C}^{4+}$ and $\mathrm{C}^{5+}$, and is two order of magnitude lower.

\subsection{Element-by-element CIE Cooling}

The last column in each part (A-AD) of Table 1 lists the total CIE cooling efficiency of each element as a function of temperature. Figure 3 shows the CIE cooling efficiencies of the major coolants as a function of temperature, assuming a solar metallicity. In making Figure 3, we adopt the elemental abundances for $\mathrm{C}, \mathrm{N}, \mathrm{O}, \mathrm{Mg}, \mathrm{Si}, \mathrm{S}$, and Fe reported by Asplund et al. (2005) for the photosphere of the Sun and the enhanced Ne abundance recommended by Drake \& Testa (2005). For the other elements we use the abundances reported by Grevesse $\&$ Sauval (1998). We list these abundances in Table 2.

Figure 3 shows the familiar peaks in the CIE cooling efficiency due to different elements. The low-temperature peak at $\sim 2 \times 10^{4} \mathrm{~K}$ is mainly due to hydrogen Ly $\alpha$ cooling. As the hydrogen neutral fraction becomes small, the contribution of hydrogen Ly $\alpha$ decreases. This peak is followed by peaks at $10^{5}$, $3 \times 10^{5}, 5 \times 10^{5}$, and $1.5 \times 10^{6} \mathrm{~K}$ due, respectively, to contributions of carbon, oxygen, neon, and iron. A second iron peak can be seen at $\sim 10^{7} \mathrm{~K}$. At higher temperatures cooling is dominated by thermal bremsstrahlung due to fully stripped ions. Contributions by other cooling elements are also shown in 

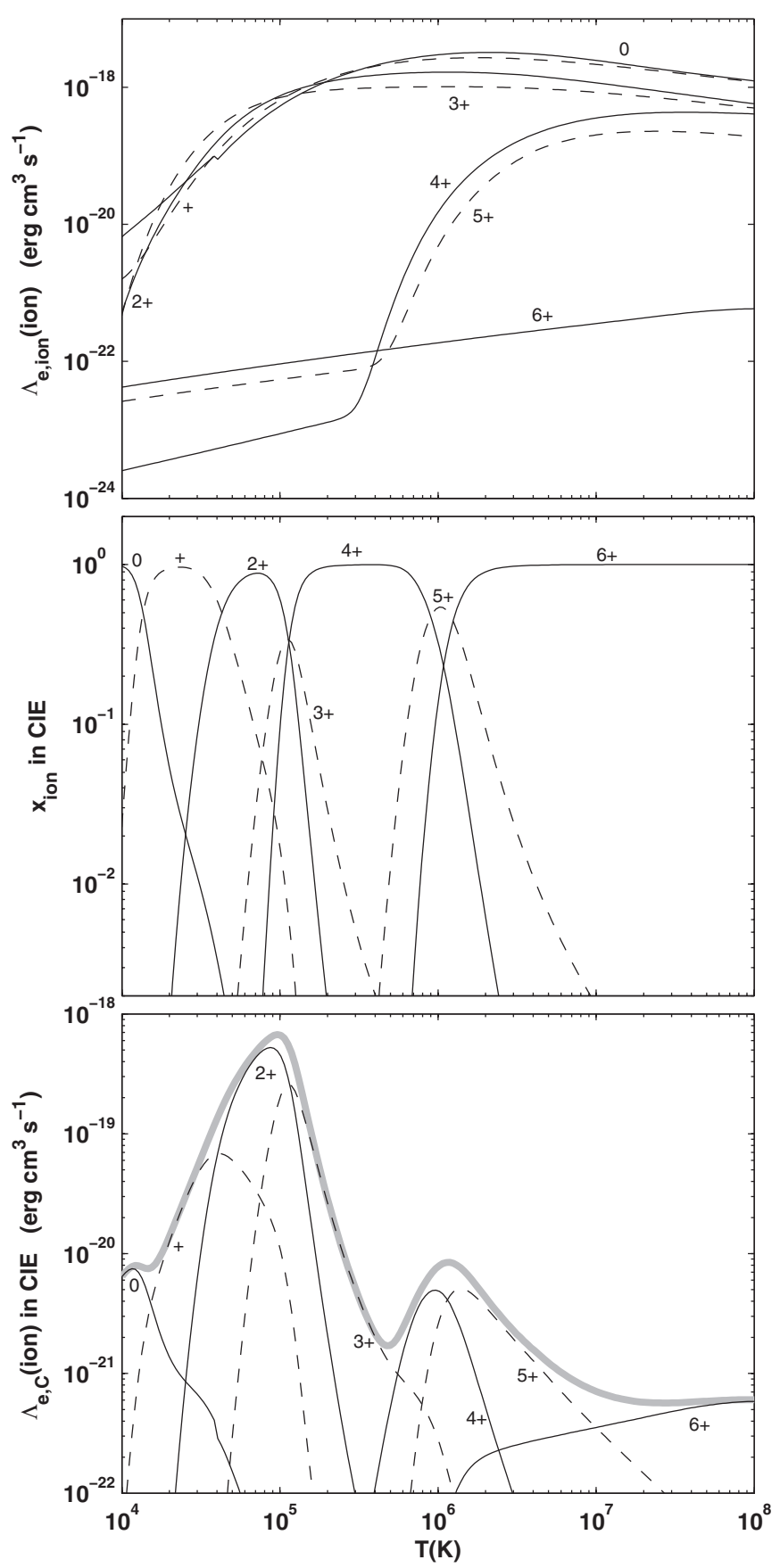

Figure 2. Cooling efficiencies of carbon ions. Upper panel: ion-by-ion cooling efficiencies for carbon ions. The cooling rate per unit volume is given by $n_{e} n_{\text {ion }} \Lambda_{e}$, ion (ion). Middle panel: carbon CIE ion fractions. Lower panel: carbon CIE cooling efficiencies for carbon ions, $x_{\text {ion }} \Lambda_{e}$, ion. The cooling rate per unit volume is given by $n_{e} n_{\mathrm{C}} \Lambda_{e, \mathrm{C}}$ (ion). The sum of CIE cooling efficiencies by all carbon ions is shown by the thick gray curve.

Figure 3. For example, cooling due to helium peaks at a temperature of $\sim 8 \times 10^{4} \mathrm{~K}$. For solar metallicity gas the helium contribution to the total cooling is small compared with that of metal-line cooling. However, at subsolar metallicities the relative contribution of helium is larger. Nitrogen, magnesium, silicon, and sulfur also contribute to the cooling below $\sim 5 \times 10^{6} \mathrm{~K}$. The results for the elemental CIE cooling efficiencies are in qualitative agreement with previous computations (e.g., Sutherland \& Dopita 1993). Differences in detail are mainly due to differences in assumed atomic data (see Gnat \& Sternberg 2007), affecting both the CIE ion fractions and the cooling efficiencies of specific cooling processes.

The upper thick gray curve shows the total contribution of all elements to the CIE, solar metallicity cooling efficiency. These results are identical to the cooling efficiencies computed by Cloudy (version 10.00) assuming CIE and a solar composition (including all elements). For comparison, the dotted curve shows the CIE cooling efficiencies of Gnat \& Sternberg (2007), which were computed using the cooling function included in Cloudy version 06.02. The agreement is excellent for $T \lesssim 2 \times 10^{6} \mathrm{~K}$, but some differences appear at higher temperatures. This is mostly due to improved treatment of the He-like iso-sequence (see Porter \& Ferland 2007) included in the newer version of Cloudy (the cooling due to He-like neon, oxygen, and magnesium was overestimated in version 06.02 ).

\section{SUMMARY}

In this paper, we present computations of the cooling efficiencies of each ion of the first 30 elements (hydrogen-zinc) individually. We use the cooling functions included in Cloudy (version 10.00) to compute the cooling efficiencies as a function of temperature, between $10^{4}$ and $10^{8} \mathrm{~K}$, assuming optically thin conditions.

The results are listed in tabular form in Table 1 (Section 3) and are available as a supplemental data file in the online version (for a guide see Table 2). For each ion, we list the cooling efficiency $\Lambda_{e \text {, ion }}(T)$ (erg $\mathrm{cm}^{3} \mathrm{~s}^{-1}$ ) as a function of temperature. The total cooling rate for any ionic composition can then be computed by multiplying the ionic efficiencies by the ion densities, $n_{e} \sum_{\text {ion }} n$ (ion) $\Lambda_{e}$, ion $\left(\mathrm{erg} \mathrm{s}^{-1} \mathrm{~cm}^{-3}\right)$.

As opposed to gas in CIE, for which the cooling efficiencies depend only on the gas temperature and metallicity, for nonequilibrium conditions the cooling efficiencies must be evaluated locally depending on the nonequilibrium ion fractions. A self-consistent computation therefore requires the collection of a large set of atomic data for all the relevant microphysical cooling processes, including numerous emission lines, thermal bremsstrahlung, and ionization and recombination processes. The results presented in this paper allow for an efficient estimate of the total cooling efficiency regardless of the ionization state.

The tables presented here use the current atomic data set within Cloudy version 10.00 . The tables provide a flexible way to access the cooling of individual species. The Cloudy atomic database is continuously updated and new versions of these tables, using the same format, will be created as the atomic data are improved. These tables will then provide easy access to future improvements as they occur.

The online tables are useful when constructing theoretical models in which nonequilibrium ionization plays a significant role and can be used, for example, in models for radiatively cooling gas, conduction fronts, fast shock waves, and turbulent mixing layers. They can also simplify the inclusion of selfconsistent nonequilibrium cooling in large-scale cosmological hydrodynamical simulations.

In Section 3.1, we present the elemental cooling efficiencies as a function of temperature for each of the first 30 elements (H-Zn) assuming CIE conditions. These results can be used to easily construct CIE cooling efficiencies for non-solar abundance ratios, as well as to estimate the cooling by elements not included in any time-dependent, nonequilibrium computations.

O.G. was supported by NASA through Chandra Postdoctoral Fellowship grant PF8-90053 awarded by the Chandra X-ray 


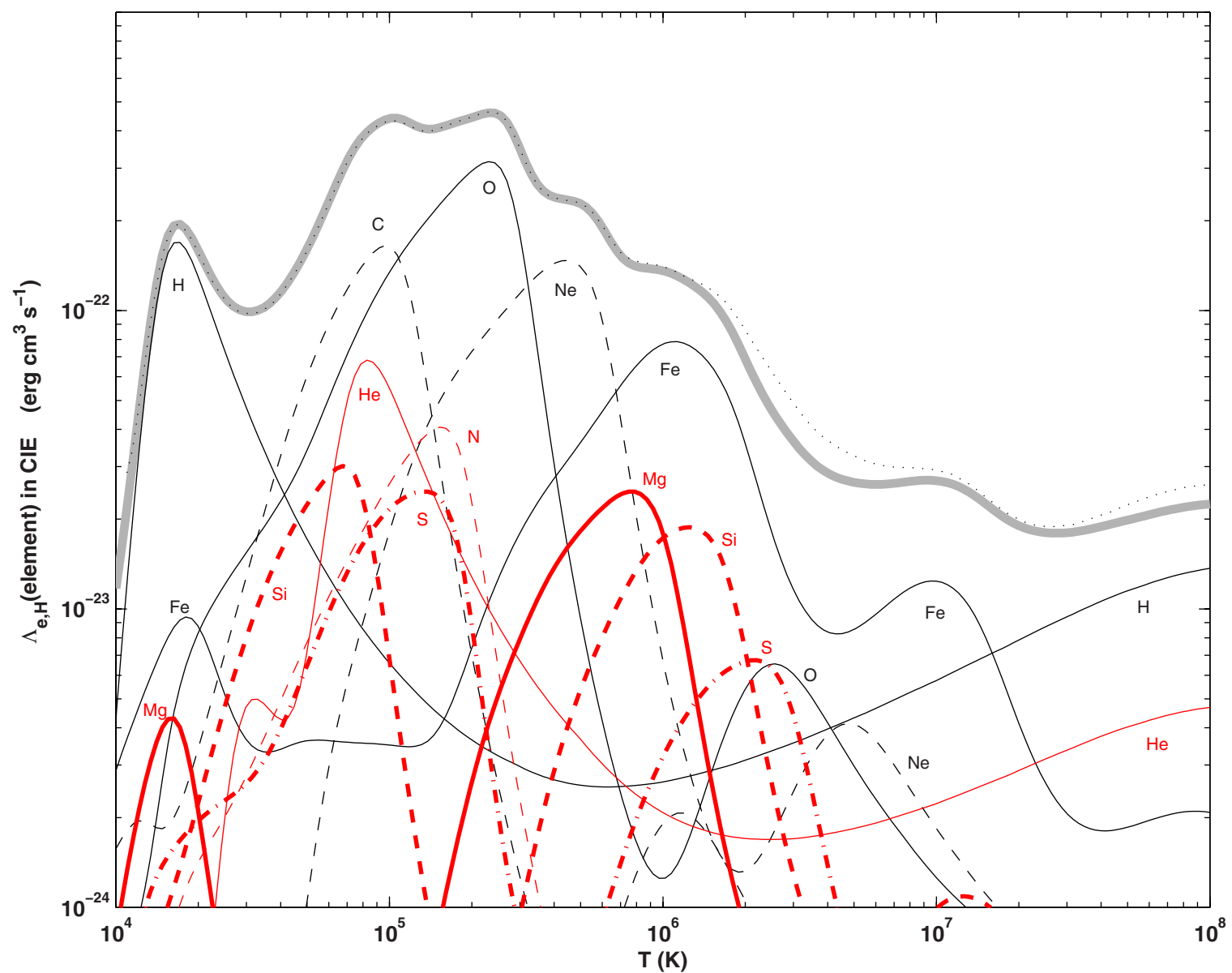

Figure 3. Element-by-element cooling efficiencies assuming CIE ion fractions and solar elemental abundance ratios (see Table 2). The total CIE cooling efficiency due to all elements is shown by the upper thick gray curve. The CIE cooling efficiency of Gnat \& Sternberg (2007), which relied on Cloudy version 06.02, is shown by the upper dotted line for comparison. The differences between the two are due to updated atomic data included in Cloudy version 10.00 .

(A color version of this figure is available in the online journal.)

Center, which is operated by the Smithsonian Astrophysical Observatory for NASA under contract NAS8-03060. Partial financial support for G.J.F's work on this project was provided by National Science Foundation grants AST 0908877 and AST 0607028, National Aeronautics and Space Administration grant 07-ATFP07-0124, and HST Theory grant AR 12125.01.

\section{REFERENCES}

Akahori, T., \& Yoshikawa, K. 2010, PASJ, 62, 335

Allen, J. W., \& Dupree, A. K. 1969, ApJ, 155, 27

Allen, M. G., Groves, B. A., Dopita, M. A., Sutherland, R. S., \& Kewley, L. J. 2008, ApJS, 178, 20

Arnaud, M., \& Rothenflug, R. 1985, A\&AS, 60, 425

Asplund, M., Grevesse, N., \& Sauval, A. J. 2005, in ASP Conf. Ser. 336, Cosmic Abundances as Records of Stellar Evolution and Nucleosynthesis, ed.

T. G. Barnes, III \& F. N. Bash (San Francisco, CA: ASP), 25

Ballantyne, D. R., Ferland, G. J., \& Martin, P. G. 2000, ApJ, 536, 773

Benjamin, R. A., Benson, B. A., \& Cox, D. P. 2001, ApJ, 554, L225

Boehringer, H., \& Hensler, G. 1989, A\&A, 215, 147

Borkowski, K. J., Balbus, S. A., \& Fristrom, C. C. 1990, ApJ, 355, 501

Cen, R., \& Fang, T. 2006, ApJ, 650, 573

Cen, R., \& Ostriker, J. P. 2006, ApJ, 650, 560

Cox, D. P., \& Tucker, W. H. 1969, ApJ, 157, 1157

Dalgarno, A., \& McCray, R. A. 1972, ARA\&A, 10, 375
Drake, J. J., \& Testa, P. 2005, Nature, 436, 525

Edgar, R. J., \& Chevalier, R. A. 1986, ApJ, 310, L27

Ferland, G. J., Korista, K. T., Verner, D. A., et al. 1998, PASP, 110, 761

Gaetz, T. J., \& Salpeter, E. E. 1983, ApJS, 52, 155

Gnat, O., \& Sternberg, A. 2007, ApJS, 168, 213

Gnat, O., \& Sternberg, A. 2009, ApJ, 693, 1514

Gnat, O., Sternberg, A., \& McKee, C. F. 2010, ApJ, 718, 1315

Graney, C. M., \& Sarazin, C. L. 1990, ApJ, 364, 561

Grevesse, N., \& Sauval, A. J. 1998, Space Sci. Rev., 85, 161

House, L. L. 1964, ApJS, 8, 307

Jordan, C. 1969, MNRAS, 142, 501

Kafatos, M. 1973, ApJ, 182, 433

Landi, E., \& Landini, M. 1999, A\&A, 347, 401

Oppenheimer, B. D., \& Davé, R. 2009, MNRAS, 395, 1875

Osterbrock, D. E., \& Ferland, G. J. (ed.) 2006, Astrophysics of Gaseous Nebulae and Active Galactic Nuclei (2nd ed.; Sausalito, CA: Univ. Science Books)

Porter, R. L., \& Ferland, G. J. 2007, ApJ, 664, 586

Raymond, J. C., Cox, D. P., \& Smith, B. W. 1976, ApJ, 204, 290

Schmutzler, T., \& Tscharnuter, W. M. 1993, A\&A, 273, 318

Schure, K. M., Kosenko, D., Kaastra, J. S., Keppens, R., \& Vink, J. 2009, A\&A, 508,751

Shapiro, P. R., \& Moore, R. T. 1976, ApJ, 207, 460

Shull, J. M., \& van Steenberg, M. 1982, ApJS, 48, 95

Sutherland, R. S., \& Dopita, M. A. 1993, ApJS, 88, 253

Slavin, J. D., Shull, J. M., \& Begelman, M. C. 1993, ApJ, 407, 83

Smith, R. K., Krzewina, L. G., Cox, D. P., Edgar, R. J., \& Miller, W. W. I. 1996, ApJ, 473, 864

Tucker, W. H., \& Gould, R. J. 1966, ApJ, 144, 244 